\title{
A double-blind, placebo-controlled clinical trial to assess the effects of a combined nutraceutical on endothelial function in patients with mild-to-moderate hypercholesterolaemia
}

\author{
Valerio Pecchioli ${ }^{1}$, Arrigo F.G. Cicero², Nazareno Lomartire ${ }^{3}$, Maria P. Gemmiti ${ }^{4}$, \\ Antonello Colangeli ${ }^{5}$, Rosanna Germanò ${ }^{6}$, Maria Pia Corsi ${ }^{7}$, Giuseppe I.W. Germanò ${ }^{6}$
}

\author{
${ }^{1}$ Cardiovascular Prevention Unit, Hospital Department ASL Frosinone, Italy \\ ${ }^{2}$ Department of Medical and Surgical Sciences, Alma Mater Studiorum University \\ of Bologna, Italy \\ ${ }^{3}$ Anaesthesiology and Intensive Care Unit, ASL, Frosinone, Italy \\ ${ }^{4}$ Cardiology and Cardiological Intensive Care Unit, ASL Frosinone, Italy \\ ${ }^{5}$ Pneumology Unit, Department of Internal Medicine, University of L'Aquila, Italy \\ "Policlinico Umberto I University "La Sapienza”, Rome, Italy \\ ${ }^{7}$ Cardiology and Cardiological Intensive Care Unit, ASL Frosinone, Italy
}

Submitted: 17 March 2020

Accepted: 18 April 2020

Arch Med Sci Atheroscler Dis 2020; 5: e36-e42

DOI: https://doi.org/10.5114/amsad.2020.94986

Copyright $\odot 2020$ Termedia \& Banach

\begin{abstract}
Introduction: There is growing interest in lipid-lowering nutraceuticals; however, there are a relative scarcity of data on combined compounds. This study was aimed to assess the efficacy and tolerability of a combined nutraceutical (CARDIOL ${ }^{\oplus}$ Forte - CF) containing polyunsaturated fatty acids, hydroxytyrosol, Coenzyme Q10, folic acid, $\mathrm{B}_{12}$ and E vitamins, piperine, and red yeast rice in patients with mild-to-moderate hypercholesterolaemia.

Material and methods: In this single-centre, double-blinded, placebo-controlled study enrolled subjects who were randomised to receive the tested combined nutraceutical for 16 weeks (CF group) or placebo (control group), in association with a low-fat diet. After 8 weeks of treatment, all patients underwent a 15-day washout period; then, a further 8 weeks of treatment was planned.

Results: Of 80 enrolled subjects, 37 completed the study in the CF group and 38 in the control group. After 8 weeks of treatment, low-density lipoprotein cholesterol levels were reduced by $17 \%$ in the CF group and by $6.4 \%$ in the control group, compared to baseline $(p=0.0001)$; these changes were improved at the end of study. Total cholesterol and triglyceride levels significantly decreased during treatment; high-density lipoprotein cholesterol did not change. In the CF group, flow-mediated dilation increased by $18.8 \%$ after 8 weeks and by $39.3 \%$ at the end of treatment. No adverse events or musculoskeletal disorders were reported in either group.

Conclusions: The tested combined nutraceutical, in association with a controlled diet, can reduce cholesterol levels and improve endothelial function, thus reducing the cardiovascular risk in patients with mild-to-moderate hypercholesterolaemia.
\end{abstract}

Key words: nutraceutical, hypercholesterolaemia, red yeast rice, monacolin K, low-density lipoprotein-cholesterol, endothelial function.

\author{
Corresponding author: \\ Prof. Arrigo F.G. Cicero \\ Department of Medical \\ and Surgical Sciences \\ Alma Mater Studiorum \\ University of Bologna \\ 40138 Bologna, Italy \\ Phone: +39 3498558017 \\ E-mail: arrigo.cicero@unibo.it
}




\section{Introduction}

Although several programs and health initiatives have been started to diminish the prevalence of modifiable risk factors for cardiovascular disease (CVD), cardiovascular mortality remains the leading cause of death worldwide [1]. A correct diet and healthy life habits, which contribute to optimising the lipid profile, form the most effective intervention to limit the onset and progression of the atherosclerotic process [2]. However, they are often not enough to achieve low-density lipoprotein cholesterol (LDL-C) levels that are recommended as therapeutic targets, and pharmacological treatments often become necessary. Many molecules with antioxidant and anti-inflammatory effects acting on the atherosclerotic process are currently available on the market and contribute to reducing cardiovascular mortality and the burden of the disease $[3,4]$. However, some of them, including statins, are associated with adverse events, which prevent their prescription or determine poor adherence to treatment [5].

In recent years, nutraceutical pharmacology has identified many food components with antioxidant and anti-inflammatory activity, with pleiotropic effects able to reduce plasma cholesterol levels and regulate lipid metabolism [6-8].

CARDIOL $^{\oplus}$ Forte is dietary supplement containing eicosapentaenoic acid (EPA) and docosahexaenoic acid (DHA), hydroxytyrosol polyphenol of olive oil, coenzyme Q10, folic acid, $\mathrm{B}_{12}$ and $\mathrm{E}$ vitamins, piperine, and monacolin $\mathrm{K}(10 \mathrm{mg})$ from red yeast rice (RYR), whose components have demonstrated to have lipid-lowering action and/or endothelial protective action [9-14].

Evidence from a meta-analysis including more than 9600 people indicated that different RYR reduced total cholesterol (TC), LDL-C, and triglycerides (TG) and increased high-density lipoprotein (HDL-C) levels. These effects were similar to those reported for statins: pravastatin, simvastatin, lovastatin, atorvastatin or fluvastatin. Furthermore, RYR monacolin K preparations were superior to nicotine and fish oils in controlling lipid metabolism, but comparable or inferior to fenofibrate and gemfibrozil [15]. Although some concerns on RYR safety in terms of musculoskeletal disorders (MuD) have been reported, a recent meta-analysis on 53 randomised studies did not evidence any association between RYR monacolin $\mathrm{K}$ and an increased risk of MuD, non- musculoskeletal disorders (nonMuD) and serious adverse events. Therefore, monacolin $\mathrm{K}$ could be considered as safe in patients with moderate hypercholesterolaemia $[16,17]$.

Reducing plasma cholesterol levels improves also the endothelial function, which is involved in development and progression of the atherosclerotic plaque [18-20]. Some evidence indicates that hydroxytyrosol and coenzyme Q10 improved endothelial function and reduced CV risk in hyperlipidaemic patients in primary prevention [21-24].

This double-blinded, placebo-controlled study aimed to assess the efficacy and tolerability of CARDIOL $^{\oplus}$ Forte in patients with mild-to-moderate hypercholesterolaemia as primary prevention [25], to evaluate the effects on endothelial function, and to describe the safety profile of monacolin $\mathrm{K}$ supplementation.

\section{Material and methods}

A single-centre, double blinded, placebo-controlled study was performed at the Cardiovascular Prevention Unit - Hospital Department ASL Frosinone, Italy on consecutive patients with mildto-moderate hypercholesterolaemia. Inclusion criteria were: i) age range from 18 to 70 years; ii) LDL-C levels $130-160 \mathrm{mg} / \mathrm{dl}$, confirmed in two consecutive examinations. Exclusion criteria were: i) the presence of cardiovascular diseases; ii) triglyceride levels > $400 \mathrm{mg} / \mathrm{dl}$; iii) use of lipid-lowering agents or drugs that modified lipid metabolism; known thyroid, liver, kidney or muscle disease; atrial fibrillation, bigeminal and trigeminal extra-systolic ventricular or supra-ventricular pulse. All patients were asked to sign an informed consent form before the enrolment. The study was approved by the local Ethics Committee (Comitato Etico Locale Lazio 2) and was performed according to the Helsinki Declaration.

During a screening phase, patients underwent a clinical-instrumental examination, in which anthropometric parameters, echocardiography, colour Doppler echocardiography, colour Doppler echocardiography on supra-aortic vessels, transaminases, creatine phosphokinase, renal function - creatinine and azotemia - HDL-C, LDL-C, triglycerides, fasting glycaemia, and glycated haemoglobin were recorded. At baseline before randomisation, flow-mediated dilatation (FMD) of the brachial artery was measured by echo-Doppler, as per the International Brachial Artery Reactivity Task Force $[26,27]$. The measurement was performed in the morning, after 12-hour fasting, always by the same operator during the study.

After the enrolment, patients were randomised to receive CARDIOL ${ }^{\oplus}$ Forte for a total of 16 weeks (CF group) or placebo (control group) (both kindly furnished by U.G.A. Nutraceuticals, Monza, MB, Italy), in combination with a diet with low lipidic content $(2000 \mathrm{kcal}, 55 \%$ carbohydrates, $20 \%$ proteins, and $25 \%$ lipids): after 8 weeks of treatment, all patients underwent a 15-day wash-out period, then a further eight weeks of treatment were planned.

Blood samples were collected at baseline, after 8 weeks of treatment (visit 1), at the end of the 
wash-out period (visit 2), and at the end of the study (visit 3). During the scheduled visits, adherence to diet, treatment efficacy, and duration of response were evaluated in both groups; tolerability was monitored throughout the study, reporting all minimal changes in enzymatic levels that could suggest any eventual MuD and non-MuD occurrence.

\section{Statistical analysis}

Primary endpoints were to determine the change in total cholesterol, LDL-C, HDL-C, and triglycerides of nutraceutical supplement and to evaluate its effect on endothelial function. Hepatic, renal, and muscular tolerability was specifically evaluated $[28,29]$.

Considering an error $\alpha$ as 0.05 and an error $\beta$ as 0.20 , and a statistical power as 0080, at least 71 patients should be included in this analysis. Clinical and demographic data and measured variables were compared using Student's test for unpaired data; the ANOVA test was used to estimate the response to different treatments (i.e. for comparing the endothelial function). Data were reported as mean and standard deviation and were considered as statistically significant when $p<0.05$. Statistical analysis was performed with SSPS for Windows version 21.0.

\section{Results}

\section{Study population}

Of 80 enrolled patients, 75 completed the study (37 in the CF group and 38 in the control group), while 5 patients were excluded for protocol violations: 3 patients in the CF group for lack of adherence to treatment and 2 patients in the control group for lack of adherence to the diet. Clinical and demographic characteristics are summarised in Table I. In addition to hypercholesterolaemia, concomitant cardiovascular risk factors, including smoke, diabetes, and hypertension, were present in 64 patients: 58 patients had one more risk factor, and eight patients had two more risk factors. In the CF group, there were 24 patients with hypertension, 5 smokers, and 3 patients with diabetes; in the control group 22 patients had hypertension and one diabetes, while 11 were smokers.

\section{Lipid profile}

After 8 weeks of treatment (visit 1), LDL-C levels were reduced by $17 \%$ in the CF group and by $6.3 \%$ in the control group compared to baseline $(p=0.002)$. These changes were persistent until the end of the wash out period (visit 2), with further reduction by $23.5 \%$ in the CF group and by $14.1 \%$ in the control. At the end of the study (visit 3), LDL-C in the CF group was reduced by $34.3 \%$, while in the control group by $22.6 \%(p=0.013)$. Furthermore, TC and TG levels were significantly decreased throughout the study in the CF group, while in the control group TC was reduced by 8.5 $\pm 5.18 \%$, and no change was observed in TG levels $(p=0.522)$ (Figure 1). HDL-C did not change in both groups (Table II).

\section{Flow-mediated dilatation}

In the CF group, FMD increased by $18.8 \pm 3.2 \%$ after 8 weeks of treatment, and a further increase was observed on visit 2 by $22.9 \pm 4.4 \%$ and on visit 3 by $39.3 \pm 5.2 \%$; in the control group, FMD increased by $10.9 \pm 4.2 \%$ on visit 1 and by $17.1 \pm 5.2 \%$ on visit 3. The increases in FMD recorded within each group are statistically significant (Table III).

\section{Tolerability}

No occurrence of MuD, non-NuD, or serious adverse events were reported during the study.

Table I. Main baseline demographic and clinical characteristics of two groups of treatment

\begin{tabular}{|c|c|c|c|c|c|}
\hline \multirow[t]{2}{*}{ Parameter } & \multicolumn{2}{|c|}{$\begin{array}{c}\text { CF group } \\
(N=37 ; 23 \text { women })\end{array}$} & \multicolumn{2}{|c|}{$\begin{array}{c}\text { Control group } \\
(N=38 ; 21 \text { women })\end{array}$} & \multirow[t]{2}{*}{$P$-value } \\
\hline & Mean & SD & Mean & SD & \\
\hline Age [years] & 57.3 & 9.3 & 54.3 & 9.8 & 0.178 \\
\hline BMI $\left[\mathrm{kg} / \mathrm{m}^{2}\right]$ & 32.8 & 5.1 & 28.5 & 4.1 & 0.561 \\
\hline $\mathrm{TC}[\mathrm{mg} / \mathrm{dl}]$ & 217.6 & 14.1 & 221.6 & 14.1 & 0.222 \\
\hline $\mathrm{HDL}-\mathrm{C}[\mathrm{mg} / \mathrm{dl}]$ & 58.7 & 14.3 & 58.3 & 13.8 & 0.899 \\
\hline LDL-C [mg/dl] & 156.3 & 30.8 & 153.5 & 32.2 & 0.064 \\
\hline TG $[\mathrm{mg} / \mathrm{dl}]$ & 130.8 & 65.3 & 117.8 & 33.8 & 0.286 \\
\hline Glucose [mg/dl] & 93.9 & 13.1 & 97.3 & 11.3 & 0.479 \\
\hline Glycated hemoglobin (\%) & 5.4 & 0.3 & 5.4 & 0.4 & 1.000 \\
\hline
\end{tabular}

$B M I$ - body mass index, TC - total cholesterol, HDL-C - high-density lipoprotein-cholesterol, LDL-C - low-density lipoprotein-cholesterol, $T G$ - triglycerides. 
In the CF group, CPK levels decreased by $7.8 \%$ after eight weeks of treatment on visit 1 and by $13.2 \%$ on visit 2 ; a small increase was observed on visit 3 (by $6.2 \%$ ) compared to visit 2 . There was no significant difference between the two groups in all visits (Table IV). AST and ALT were reduced in both groups, while creatinine did not change (Table V).

\section{Discussion}

There is growing interest in lipid-lowering nutraceuticals; however, there are a relative scarcity of data on combined compounds and on their effect on vascular function [30].

This double-blinded, placebo-controlled study showed that the tested combined product had superior efficacy to placebo in reducing LDL-C levels in patients with mild-to-moderate hypercholesterolaemia in primary prevention after 16 weeks of treatment. Our results were consistent with literature evidence [31, 32].

An early LDL-C reduction was observed after 8 weeks of treatment, and it was enhanced at

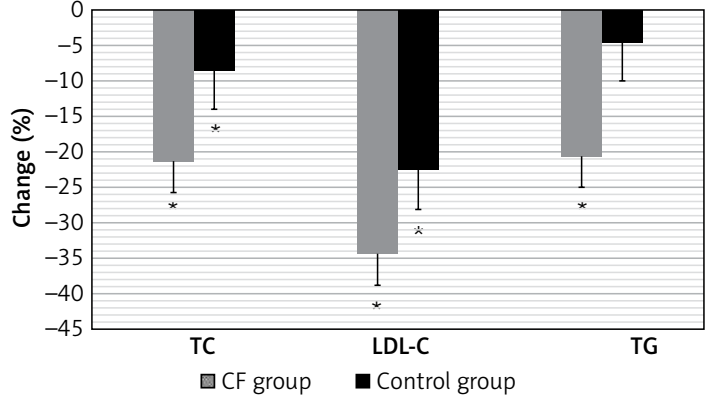

Figure 1. Change in total cholesterol, low-density lipoprotein - cholesterol and triglycerides in CF and control groups at the end of the study (visit 3)

${ }^{\star} P<0.005$.

the end of washout period, thus indicating a persistent and durable response compared to diet alone. The tested combined product improved also the endothelial function, as documented by a significant FMD increase, in all study visits. This is of particular interest because a meta-analysis of 35 studies including 17,280 subjects showed that each $1 \%$ increase in FMD is associated with a $12 \%$

Table II. Total cholesterol, HDL-C, LDL-C and triglycerides levels in CF and control groups and change from baseline to each study visit

\begin{tabular}{|c|c|c|c|c|c|}
\hline Parameter & CF group & Control group & $\begin{array}{l}\text { Change \% in CF } \\
\text { groups vs. baseline }\end{array}$ & $\begin{array}{l}\text { Change } \% \text { in Control } \\
\text { group vs. baseline }\end{array}$ & $P$-value \\
\hline \multicolumn{6}{|l|}{ TC: } \\
\hline TO & $217.6 \pm 14.1$ & $221.6 \pm 14.1$ & & & 0.223 \\
\hline T1 & $182.9 \pm 24.7$ & $188.0 \pm 20.0$ & 16.1 & 15.1 & 0.0001 \\
\hline $\mathrm{T} 2$ & $177.5 \pm 22.7$ & $184.8 \pm 21.4$ & 18.4 & 16.6 & 0.038 \\
\hline $\mathrm{T} 3$ & $171.1 \pm 21.4$ & $202.8 \pm 20.0$ & 21.3 & 8.5 & 0.007 \\
\hline \multicolumn{6}{|l|}{ HDL-C: } \\
\hline TO & $58.7 \pm 14.2$ & $58.3 \pm 13.8$ & & & 0.899 \\
\hline T1 & $57.7 \pm 11.9$ & $55.1 \pm 10.9$ & 1.7 & 5.5 & 0.334 \\
\hline $\mathrm{T} 2$ & $55.8 \pm 12.1$ & $53.6 \pm 10.5$ & 4.9 & 8.1 & 0.401 \\
\hline $\mathrm{T} 3$ & $55.1 \pm 9.9$ & $59.1 \pm 11.5$ & 6.3 & -1.4 & 0.104 \\
\hline \multicolumn{6}{|l|}{ LDL-C: } \\
\hline TO & $156.5 \pm 32.2$ & $152.3 \pm 30.8$ & & & 0.060 \\
\hline $\mathrm{T} 1$ & $129.8 \pm 11.6$ & $142.7 \pm 10.8$ & 17.0 & 6.3 & 0.002 \\
\hline $\mathrm{T} 2$ & $119.7 \pm 16.9$ & $130.7 \pm 15.3$ & 23.5 & 14.1 & 0.004 \\
\hline T3 & $102.7 \pm 14.1$ & $117.8 \pm 13.8$ & 34.3 & 22.6 & 0.013 \\
\hline \multicolumn{6}{|c|}{ Triglycerides: } \\
\hline TO & $130.7 \pm 65.3$ & $117.8 \pm 33.8$ & & & 0.284 \\
\hline T1 & $115.5 \pm 48.8$ & $108.7 \pm 31.6$ & 11.6 & 7.7 & 0.476 \\
\hline $\mathrm{T} 2$ & $110.9 \pm 44.7$ & $110.7 \pm 33.9$ & 15.1 & 6.0 & 0.983 \\
\hline T3 & $103.7 \pm 45.2$ & $112.4 \pm 37.9$ & 20.6 & 4.6 & 0.368 \\
\hline
\end{tabular}


Table III. Baseline flow-mediated dilatation and change after treatment

\begin{tabular}{|lcccccc|}
\hline FMD & CF group & Change \% & $P$-value & Control group & Change $\%$ & $P$-value \\
\hline T0 & $12.21 \pm 0.03$ & & $11.12 \pm 0.04$ & & \\
\hline T1 & $14.53 \pm 0.04$ & $18.8 \pm 3.2$ & & $12.35 \pm 0.04$ & $10.9 \pm 4.2$ & \\
\hline T2 & $15.02 \pm 0.04$ & $22.9 \pm 4.4$ & & $13.14 \pm 0.04$ & $18.0 \pm 4.1$ & \\
\hline T3 & $17.06 \pm 0.05$ & $39.3 \pm 5.2$ & $<0.001$ & $13.05 \pm 0.03$ & $17.1 \pm 5.2$ & 0.004 \\
\hline
\end{tabular}

Table IV. Changes of creatinine phosphokinase levels

\begin{tabular}{|lcccccc|}
\hline Parameter & \multicolumn{3}{c}{ NC group } & \multicolumn{3}{c|}{ Control group } \\
\cline { 2 - 7 } & Mean & SD & Change \% & Mean & SD & Change \% \\
\hline T0 & 113.60 & 55.13 & & 114.32 & 36.79 & \\
\hline T1 & 104.72 & 37.02 & 7.81 & 104.13 & 44.21 & 8.91 \\
\hline T2 & 98.61 & 33.91 & 13.19 & 102.46 & 43.82 & 10.37 \\
\hline T3 & 104.95 & 43.95 & 7.61 & 110.15 & 43.02 & -3.64 \\
\hline
\end{tabular}

Table V. Renal and hepatic tolerability

\begin{tabular}{|lcccccc|}
\hline Parameter & \multicolumn{3}{c}{ CF group } & \multicolumn{3}{c|}{ Control group } \\
\cline { 2 - 6 } & Baseline (T0) & $\begin{array}{c}\text { End of } \\
\text { treatment (T3) }\end{array}$ & $P$-value & Baseline (T0) & $\begin{array}{c}\text { End of } \\
\text { treatment (T3) }\end{array}$ & $P$-value \\
\hline AST & $19.83 \pm 4.11$ & $17.69 \pm 2.96$ & 0.012 & $21.17 \pm 5.76$ & $17.54 \pm 2.89$ & 0.001 \\
\hline ALT & $19.91 \pm 6.12$ & $17.74 \pm 5.31$ & 0.109 & $22.97 \pm 7.96$ & $18.21 \pm 4.47$ & 0.003 \\
\hline Creatinine & $0.78 \pm 0.11$ & $0.79 \pm 0.12$ & 0.699 & $0.85 \pm 0.13$ & $0.82 \pm 0.17$ & 0.345 \\
\hline
\end{tabular}

$A S T$ - aspartate aminotransferase, $A L T$ - alanine aminotransferase.

reduction in predicted cardiovascular events (relative risk $=0.88,95 \% \mathrm{Cl}: 0.84-0.91, p<0.001)$ $[33,34]$.

Overall, these results were mainly related to pharmacokinetic and pharmacodynamic characteristics of nutraceutical supplement. Indeed, the tested combined product contains molecules that have different mechanisms of actions and synergistically act to globally reduce the cardiovascular risk in the CF group. Monacolin $\mathrm{K}$ inhibits HMGCoA reductase, omega- 3 EPA and DHA fatty acids reduce triglyceride synthesis, and the concomitant administration of vitamin E, coenzyme Q10, and hydroxytyrosol significantly reduces cholesterol levels and LDL-C oxidation, thus ameliorating endothelial-dependent vasodilation in the CF group compared to controls.

RYR alone or in combination with monacolin $\mathrm{K}$ demonstrated a favourable safety profile; in their meta-analysis, Fogacci et al. did not find statistical increased probability of the occurrence of adverse events [17]. Consistently, our data showed that monacolin $\mathrm{K}$ did not determine any MuD or non-MuD, as suggested by normal levels of CPK and normal hepatic and renal functioning in both study groups.
The study has some limitations. The population size was relatively small but adequately powered. Moreover, the study design - randomised and double-blinded - and treatment duration allowed us to describe a difference among treatment and control groups. Some patients included in the analysis were under anti-hypertensive therapy, which could affect endothelial function. However, in all cases the therapy was well established and ongoing before the enrolment; therefore, it could not be considered as a relevant cofounding factor in FMD assessment. Finally, the test of a combined compound does not allow us to draw conclusions about the responsibility of a single component as regards the observed effects. However, data literature already confirmed the effect of the single nutraceutical components, while we aimed to test the efficacy and tolerability of the tested combined product.

In conclusion, a nutraceutical supplement containing lipid-lowering and antioxidant components, in association with a lifestyle change and a controlled diet, can significantly reduce cholesterol levels and improve endothelial function, thus reducing the cardiovascular risk in patients with mild-to-moderate hypercholesterolaemia. 


\section{Conflict of interest}

The authors declare no conflict of interest.

\section{References}

1. World Health Organization. Media Centre. Cardiovascular Diseases (CVDs) 2012. Fact Sheet Nu317 September. Available: http://www.who.int/mediacentre/factsheet/ fs317. Last accessed: January 6th, 2020.

2. Ross R. The pathogenesis of atherosclerosis: a perspective for the 1990s. Nature 1993; 362: 801-9.

3. Cooney MT, Dudina A, Whincup P, et al. Re-evaluating the Rose approach: comparative benefits of the population and high-risk preventive strategies. Eur J Cardiovasc Prev Rehabil 2009; 16: 541-9.

4. Bjorck L, Rosengren A, Bennett K, Lappas G, Capewell S. Modelling the decreasing coronary heart disease mortality in Sweden between 1986 and 2002. Eur Heart J 2009; 30: 1046-56.

5. Kamal-Bahl SJ, Burke T, Watson D, Wentworth C. Discontunuation of lipid modifying drugs among commercially insured United States patients in recent clinical practice. Am J Cardiol 2007; 99: 530-4.

6. Derosa G, Catena G, Raddino R, et al. Effects on oral fat load of a nutraceutical combination of fermented red rice, sterol esters and stanols, curcumin, and olive polyphenols: a randomized, placebo-controlled trial. Phytomedicine 2018; 42: 75-82.

7. Cicero AF, Colletti A, Fogacci F, Bove $M$, Rosticci $M$, Borghi C. Effects of a combined nutraceutical on lipid pattern, glucose metabolism and inflammatory parameters in moderately hypercholesterolemic subjects: a double-blind, crossover, randomized clinical trial. High Blood Press Cardiovasc Prev 2017; 24: 13-8.

8. Marazzi G, Campolongo G, Pelliccia F, et al. Comparison of low-dose statin versus low dose statin + Armolipid Plus in high-intensity statin-intolerant patients with a previous coronary event and percutaneous coronary intervention (ADHERENCE trial). Am J Cardiol 2017; 120: 893-7.

9. Kayla RZ, Walker MK. Omega-3 polyunsaturated fatty acids improve endothelial function in humans at risk for atherosclerosis: a review. Prostaglandins Other Lipid Mediat 2018; 134: 131-40.

10. Vilaplana-Pérez C, Auñón D, García-Flores LA, Gil-Izquierdo A. Hydroxytyrosol and potential uses in cardiovascular diseases, cancer, and AIDS. Front Nutr 2014; 1: 18.

11. Gao L, Mao Q, Cao J, Wang Y, Zhou X, Fan L. Effects of coenzyme Q10 on vascular endothelial function in humans: a meta-analysis of randomized controlled trials. Atherosclerosis 2012; 221: 311-6.

12. Cicero AFG, Colletti A, Bajraktari G, et al. Lipid-lowering nutraceuticals in clinical practice: position paper from an International Lipid Expert Panel. Nutr Rev 2017; 75: 731-67.

13. Cicero AF, Morbini M, Rosticci M, D”Addato S, Grandi E, Borghi C. Middle-term dietary supplementation with red yeast rice plus coenzyme Q10 improves lipid pattern, endothelial reactivity and arterial stiffness in moderately hypercholesterolemic subjects. Ann Nutr Metab 2016; 68: 213-9.

14. Badmaev V, Majeed M, Prakash L. Piperine derived from black pepper increases the plasma levels of coenzyme Q10 following oral supplementation J Nutr Biochem 2000; 11: 109-13.
15. Liu J, Zhang J, Shi Y, Grimsgaard S, Alraek T, Fønnebø V. Chinese red yeast rice (Monascus purpureus) for primary hyperlipidemia: a meta-analysis of randomized controlled trials. Chin Med 2006; 1: 4.

16. Younes M, Aggett P, Aguilar F, et al.; ESFA Panel on Food Additives and Nutrient Sources added to Food (ANS). Scientific opinion on the safety of monacolins in red yeast rice. EFSA J 2018; 16: 5368-73.

17. Fogacci F, Banach M, Mikhailidis DP, et al. Safety of red yeast rice supplementation: a systematic review and meta-analysis of randomized controlled trials. Pharmacol Res 2019; 143: 1-16.

18. Endemann DH, Schiffrin EL. Endothelial dysfunction. J Am Soc Nephrol 2004; 15: 1983-92.

19. Affuso F, Ruvolo A, Micillo F, Saccà L, Fazio S. Effects of a nutraceutical combination (berberine, red yeast rice and policosanols) on lipid levels and endothelial function randomized, double-blind, placebo-controlled study. Nutr Metab Cardiovasc Dis 2010; 20: 656-61.

20. Henry PD, Cabello OA, Chen CH. Hypercholesterolemia and endothelial dysfunction. Curr Opin Lipidol 1995; 6: 190-5.

21. Martinez-Gonzalez J, Raposo B, Rodriguez C, Badimon L. 3-hydroxy-3-methylglutaryl coenzyme $A$ reductase inhibition prevents endothelial NO synthase downregulation by atherogenic levels of native LDLs: balance between transcriptional and posttranscriptional regulation. Arterioscler Thromb Vasc Biol 2001; 21: 804-9.

22. Marx W, George ES, Mayr HL, et al. Effect of high polyphenol extra virgin olive oil on markers of cardiovascular disease risk in healthy Australian adults (OLIVAUS): a protocol for a double-blind randomized, controlled, cross-over study. Nutr Diet 2019 Apr 17. doi: 10.1111/1747-0080.12531.

23. Lee BJ, Tseng YF, Yen CH, Lin PT. Effects of coenzyme Q10 supplementation (300 mg/day) on antioxidation and anti-inflammation in coronary artery disease patients during statins therapy: a randomized, placebo-controlled trial. Nutr J 2013; 12: 142.

24. Storniolo CE, Roselló-Catafau J, Pintó X, Mitjavila MT, Moreno J. Polyphenol fraction of extra virgin olive oil protects against endothelial dysfunction induced by high glucose and free fatty acids through modulation of nitric oxide and endothelin-1. Redox Biol 2014; 2: 971-7.

25. Feng SJ, Tang ZH, Wang Y, et al. Potential protective effects of red yeast rice in endothelial function against atherosclerotic cardiovascular disease. Chin J Nat Med 2019; 17: 50-8.

26. Catapano AL, Graham I, De Backer G, et al. The Task Force for the Management of Dyslipidaemias of the European Society of Cardiology (ESC) and European Atherosclerosis Society (EAS). ESC/EAS Guidelines for the Management of Dyslipidaemias. Eur Heart J 2016; 37 : 2999-3058.

27. Corretti MC, Anderson TJ, Benjamin EJ, et al. International brachial artery reactivity task force. Guidelines for the ultrasound assessment of endothelial-dependent flow-mediated vasodilation of the brachial artery: a report of the International Brachial Artery Reactivity Task Force. J Am Coll Cardiol 2002; 39: 257-65.

28. Cicero AF, Ferroni A, Ertek S. Tolerability and safety of commonly used dietary supplements and nutraceuticals with lipid-lowering effects. Expert Opin Drug Saf 2012; 11: 753-66.

29. European Society of Hypertension-European Society of Cardiology Guidelines Committee. 2003 European So- 
ciety of Hypertension-European Society of Cardiology guidelines for the management of arterial hypertension. J Hypertens 2003; 21: 1011-53.

30. Patti AM, Al-Rasadi K, Giglio RV, et al. Natural approaches in metabolic syndrome management. Arch Med Sci 2018; 14: 422-41.

31. Gerards MC, Terlou RJ, Yu H, Koks CH, Gerdes VE. Traditional Chinese lipid-lowering agent red yeast rice results in significant LDL reduction but safety is uncertain a systematic review and meta-analysis. Atherosclerosis 2015; 240: 415-23.

32. Sahebkar A, Serban MC, Gluba-Brzózka A, et al. Lipidmodifying effects of nutraceuticals: an evidence-based approach. Nutrition 2016; 32: 1179-92.

33. Cicero AF, Colletti A. Combinations of phytomedicines with different lipid lowering activity for dyslipidemia management: the available clinical data. Phytomedicine 2016; 23: 1113-8.

34. Matsuzawa Y, Kwon TG, Lennon RJ, Lerman LO, Lerman A. Prognostic value of flow-mediated vasodilation in brachial artery and fingertip artery for cardiovascular events: a systematic review and meta-analysis. J Am Heart Assoc 2015; 4: e002270. 